$\underline{\text { Review }}$

\title{
A review of hepatitis B virus infection in Sri Lanka
}

\author{
F Noordeen ${ }^{1}$, FNN Pitchai ${ }^{1}$, RA Rafeek $^{1}$ \\ Sri Lankan Journal of Infectious Diseases 2015 Vol.5 (2):42-50 \\ DOI: http://dx.doi.org/10.4038/sljid.v5i2.8087
}

\begin{abstract}
Hepatitis B virus (HBV) infection is a major public health concern in many Southeast Asian, South Asian and African countries. HBV infection is transmitted sexually or by other parenteral routes. HBV causes an acute viral hepatitis or chronic infection, largely based on the age at which the infection is acquired. In the majority of infected infants, HBV causes a chronic protracted infection for decades without symptoms. After several decades, the infection might flare up to give rise to an active hepatitis followed by sero-conversion to anti-HBs. However, the vast majority of patients with chronic HBV (CHB) infection will have chronic liver disease. Some with CHB infection develop a primary liver cancer, hepatocellular carcinoma (HCC) later in life. Approximately 25 percent of chronically infected individuals die prematurely of cirrhosis or HCC.

The prevalence of HBV infection in Sri Lanka is estimated to be less than $2 \%$. Sri Lanka is therefore considered a country of low endemicity. This prevalence rate of HBV in Sri Lanka is very different to that in many other South Asian countries such as India and Bangladesh.

Diagnosis of HBV infection in Sri Lanka is carried out in many private and state laboratories using immunochromatography based rapid assays or ELISAs for HBV surface antigen (HBsAg) detection. Those with latter stages of $\mathrm{CHB}$ are treated with currently available nucleotide / nucleoside analogues in some private sector hospitals where testing facilities for virus load during different stages of treatment is available.

Sri Lanka has a policy of immunizing healthcare workers $(\mathrm{HCW})$ who are at risk of acquiring $\mathrm{HBV}$ occupationally. Sri Lanka included HBV immunization in the Expanded Program of Immunization (EPI) with effect from 2003. In all HBsAg immunization programmes for HCW, testing for the protective anti-HBs response is mandatory in those who receive a complete course of immunization in order to ensure immunity in responders and to re-vaccinate non-responders.
\end{abstract}

Keywords: HBV, Sri Lanka, epidemiology, immune status, diagnosis

\section{Introduction}

This review compiles what we know about the status of hepatitis B virus infection in Sri Lanka, using information from published peer reviewed journal papers and abstracts from 1996 to 2015 with

${ }^{1}$ Department of Microbiology Faculty of Medicine, University of Peradeniya

Address for correspondence: Dr. Faseeha Noordeen, Department of Microbiology, Faculty of Medicine, University of Peradeniya, Sri Lanka. Telephone No: 94812396532 Email - faseeha.noordeen12@gmail.com

Received 24 May 2015 and revised version accepted 14 October 2015 
the help of PubMed and Google search engines. The global literature has been used to complete the general aspects of the disease, including virology and epidemiology.

Hepatitis B is a potentially life threatening, liver disease caused by the hepatitis B virus (HBV). The disease is a major global health problem affecting Asia, Africa, Southern Europe and Latin America and is the most serious type of viral hepatitis (Figure 1). ${ }^{1,2} \mathrm{HBV}$ endemicity can be categorized into 3 groups based on the sero-prevalence of HBV surface antigen (HBsAg) in a particular geographical area. A region is considered highly endemic if sero-prevalence exceeds $8 \%$, of intermediate endemicity if $2-8 \%$ and of low endemicity if $\leq 2 \%$, $^{1,2}$

In Sri Lanka, HBV infection is categorized as a notifiable infection and has been reported from many parts of the island. Sri Lanka has a intermediate prevalence for HBV infection with a prevalence of HBsAg positivity of less than $1 \%$ and not more than $2.5 \%$ in the general population. ${ }^{3}$ For example, according to the Epidemiology Unit, Ministry of Health data in 2010, viral hepatitis including those due to $\mathrm{HBV}$ infection has been reported from different parts of the country throughout the year. ${ }^{4}$ However, this pooled data by the Epidemiology Unit, Ministry of Health does not clearly state the number of viral hepatitis caused by HBV. Although the prevalence of HBV infection is low to intermediate in Sri Lanka, major life style changes in communities due to globalization, factors such as recreational drug use, night life, travel and social networking might lead to a relative rise in risk behaviour that might contribute to an increased spread of $\mathrm{HBV}$ infection. ${ }^{1,5,6}$

Studies carried out on the distribution of HBV infection around the world indicates that $75 \%$ percent of HBV carriers are concentrated in the Asia Pacific region ${ }^{5}$ rendering Sri Lanka a likely target for increased exposure to HBV infection. Although Sri Lanka has a low prevalence at present, given the

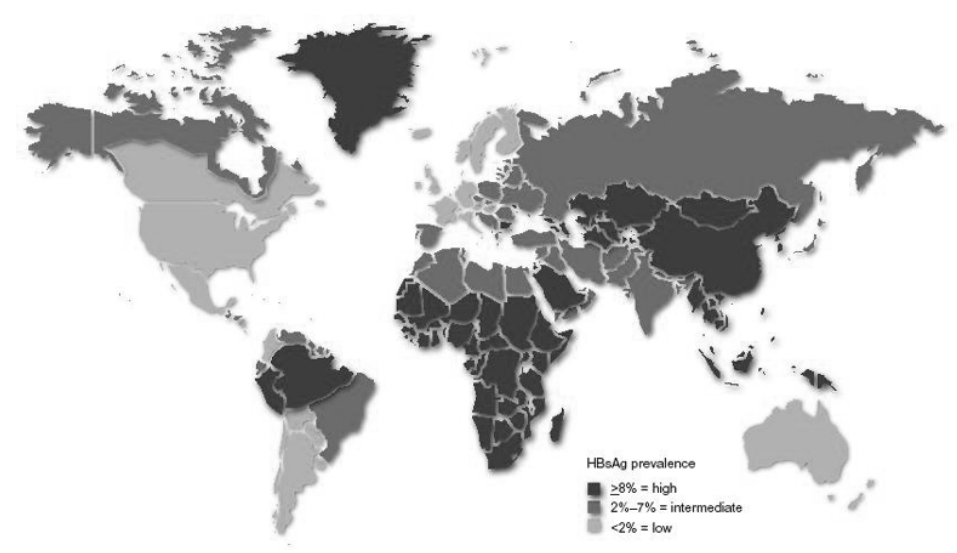

Figure 1.

Global prevalence of HBsAg positivity, Center for Disease Control, 2008. http://wwn.cdc.gov/travel/yellowbookch 4-HepB.aspx. ${ }^{36}$ current changes, there is no guarantee that the island will remain so in the future. Moreover, HBV infections are a major health problem in the South Asian Association of Regional Corporation (SAARC) region. Neighbouring countries such as India, Pakistan and Bangladesh seem to have intermediate to high prevalence rates for $\mathrm{HBV}$ infection ${ }^{6}$ predisposing Sri Lanka to risk of exposure to $\mathrm{HBV}$ through travel and entertainment. Travel between Sri Lanka and these countries is a potential contributor to increased exposure as Sri Lankans go to these countries for medical treatment, jobs, entertainment, higher education and tourism and an increasing number of foreigners come to Sri Lanka for tourism, entertainment and business.

The virus is known to be 50-100 times more infectious than human immunodeficiency virus (HIV) and much more resilient, but the most shocking aspect of $\mathrm{HBV}$ is its continuous spread despite the 
availability of a safe and effective vaccine. ${ }^{7} \mathrm{CHB}$ can be treated to control the HBV replication but treatment is expensive and the unpredictability of response to treatment makes treatment ineffective in some patients. ${ }^{3,8}$ Additionally, response to treatment needs to be monitored using authentic detection of virus load at regular intervals or at least during clinical suspicion of emergence of resistance to the antiviral in use in the patient. Overall, treatment for CHB and subsequent monitoring requires expertise, availability of currently accepted antiviral drugs (nucleotide / nucleoside analogues) and sophisticated laboratory tests, all of which require substantial funding. Such systems are not currently available in state funded hospitals in Sri Lanka. They are however, available in major private hospitals for those who can afford such treatment options.

The clinical course of CHB varies among individuals. ${ }^{8}$ Infection with HBV leads to a wide spectrum of clinical presentations, ranging from asymptomatic carrier states to acute self-limiting infection or acute fulminant hepatitis, $\mathrm{CHB}$ or $\mathrm{CHB}$ leading to cirrhosis and / or HCC and finally death. This pattern of progression shows the complexity of the disease. ${ }^{9}$ The risk of developing HCC is more than a hundred-fold in those with $\mathrm{CHB}$ compared to those without $\mathrm{CHB} .^{10}$

\section{Hepatitis B virology and transmission}

The HBV (Figure 2) is a 42-nm, spherical double-shelled virus of the family Hepadnaviridae.$^{10}$ The HBV genome consists of an incomplete double stranded DNA. Hepadnaviruses including HBV are known to have a strong affinity towards liver cells. HBV is transmitted by either percutaneous or mucosal exposure to blood or other infectious body fluids. Modes of transmission of HBV are the same as the HIV. However, unlike HIV, HBV can survive outside the body in dried blood or secretions, for at least 7 days. $^{9}$ The primary routes of HBV transmission include perinatal

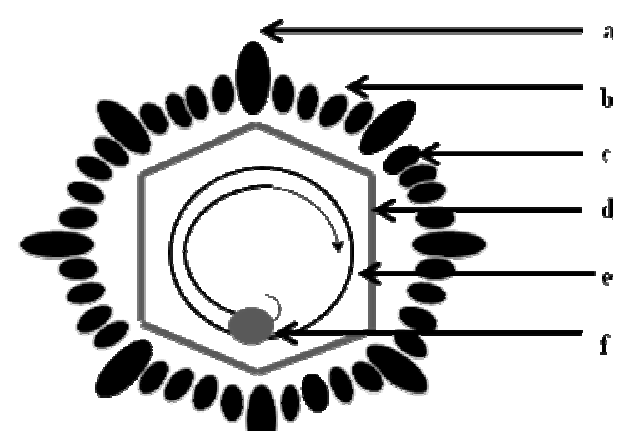

Figure 2.

Schematic diagram of HBV showing different structural components. a: large (L) surface protein; $\quad$ b: medium (M) surface protein; c: small (S) surface protein; $\quad$ a, b and c: HBV surface antigen (HBsAg); d: HBV core antigen $(\mathrm{HBcAg})$; e: partially double stranded DNA genome; f: HBV polymerase.

Adapted from Jilbert AR, Mason WS and Kann M 2008. ${ }^{10}$ transmission (from mother to baby at birth), childhood infection or inapparent infection through close interpersonal contact with infected household members, unsafe injection practices, blood transfusions and sexual contact.

Among infants in Sri Lanka, perinatal transmission from HBV infected (HBsAg-positive) mothers is the primary route of transmission. ${ }^{3}$ During childhood, child-to-child transmission through infected blood and saliva from one child to the other by either sores or breaks in the skin and from contaminated fomites account for most infections. ${ }^{11}$ Studies show that it is possible for the disease to be transmitted by sharing towels and tooth brushes. ${ }^{11}$ Sexual contact is the primary route of transmission among adolescents and adults. In addition, older age groups are at risk of transmission from contaminated needles and blood transfusions, ${ }^{8}$ although transmission through the latter is almost nil at present due to proper screening of blood. 


\section{HBV epidemiology in Sri Lanka}

The exact incidence or prevalence of HBV in the general population of Sri Lanka is unknown. Seroepidemiological studies carried out in the country indicate that the prevalence of HBsAg varies between 0.1 to $2.5 \% .^{12}$ Most studies carried out in Sri Lanka tend to concentrate on risk groups rather than the general population. According to a study carried out by Eeswaraarachchige $e t a l$ in 1993 on HBsAg carrier status and associated risk factors for acquiring HBV infection among the general population including nurses in a given district of Sri Lanka, the HBsAg carrier rate in the studied community was $2.5 \% .{ }^{13}$ The carrier rate was high (8.3\%) among children in the age group below five years. The female to male ratio of prevalence of HBsAg was 4.7:1 in this study. ${ }^{13}$ The $\mathrm{HBsAg}$ carrier state was found to be significantly associated with a history of jaundice, contact and socio-economic status of the individual. Horizontal child to child transmission of HBV infection appeared to be more common than vertical transmission and other perinatal modes of transmission. The HBsAg carrier rate among nursing officers in the study was $2 \%$ which was 2.5 times higher than the carrier rate among females in the rest of the community. ${ }^{13}$ Age, duration of employment, history of a needle prick injury and past illness with jaundice in the immediate subjects, their spouses and in other family members were found to be a major risk factor for HBV infection among the nurses. Different occupational settings, different health institutions or wards were not found to be associated with an increased risk for acquiring HBV infection. Knowledge and awareness on HBV infection was found to be inadequate in $69.3 \%$ of nursing officers. A significant number of nurses observed during the study were not following standard precautions when performing minor invasive surgical procedures on patients. Hand washing methods practiced by the nurses prior to and after handling patients did not conform to standard infection control protocols. The findings of this study justified the need for immunization against hepatitis B for children and nursing officers. Moreover, this study also highlighted the importance of an interventional program to improve knowledge and practice of nursing staff with regard to HBV transmission. ${ }^{13}$

In contrast to the findings of the previous study, ${ }^{13}$ a similar study carried out in the Colombo District (the capital of Sri Lanka) showed the relationship between HBsAg carrier status and ethnicity of the population. The study demonstrated a sero-prevalence of HBsAg of $0.46 \%$ among the Moors in the Colombo District. ${ }^{14}$ Despite the small number of HBsAg positive individuals among the Moors, they have a higher risk of contracting HBV infection due to overcrowded living conditions which may increase chances of horizontal blood contact among children, household members and neighbours. ${ }^{14}$

Sri Lankan children with thalassaemia and other children undergoing multiple transfusions for medical reasons are at a higher risk of contracting HBV infection than healthy children. ${ }^{15}$ Another study carried out at the Cancer Institute, Maharagama, Sri Lanka gives ample evidence of HBV transmission at an increased rate among cancer patients on chemotherapy, especially patients with acute lymphoblastic leukaemia. ${ }^{16}$ The same study also shows that the HBV prevalence rate is high among cancer patients undergoing multiple invasive tests and therapeutic procedures. ${ }^{16,18}$ Once infected, there is an increased possibility of the infection progressing to chronicity as the immune system is compromised in these patients. It has been observed by clinicians of the Cancer Hospital, Maharagama that there has definitely been an increase of HBV infection among cancer patients. ${ }^{18}$ This is even more likely in infected children due to their immature immune system. ${ }^{5}$ Thus the 
likelihood of horizontal transmission of the virus is high in cancer patients institutionalised for invasive investigations and therapy ${ }^{16}$ and overcrowded households ${ }^{14}$ in Sri Lanka.

A community-based HBsAg sero-prevalence study in 2003 focused on investigating the risk of perinatal transmission of HBV in the Western Province of Sri Lanka. Based on the HBsAg seroprevalence, the study concluded that the exposure of antenatal mothers to HBV was low in the Western Province of Sri Lanka. ${ }^{19,20}$ This study also showed that none of the exposed mothers had evidence of chronic HBV infection. Therefore it was concluded that perinatal infections are not an important route of HBV transmission in the Western Province of Sri Lanka. ${ }^{19,20}$

While a number of studies have been conducted on the prevalence of HBV infection, either in institutionalised patients or the general community, including nurses, in Sri Lanka, only a few studies have been carried out to determine the dominant genotype of HBV among those infected. One study carried out in Colombo by Manamperi et al $(2011)^{21}$ showed that the dominant HBV genotype was genotype B with an incidence of $36 \%$ among a cohort of CHB patients. The least prevalent among this cohort was genotype $\mathrm{A}$ at $8 \%$ while $\mathrm{C}$ and $\mathrm{D}$ occurred at a prevalence of 16 and $12 \%$, respectively. ${ }^{21}$ A more recent study carried out by Jayasuriya et al $(2015)^{22}$ in Kandy in a small cohort of HBsAg positive individuals showed the presence of genotype A. These studies collectively show the presence of multiple genotypes of HBV in Sri Lanka ${ }^{21,22}$ and this might have implications on the epidemiology of HBV infection in the future.

\section{Laboratory diagnosis of hepatitis B in Sri Lanka}

The viral marker most commonly used in the laboratory diagnosis of HBV infection in Sri Lanka as well as the rest of the world is HBsAg. Replication of the virus in hepatocytes during infection results in the release of HBsAg into the bloodstream. The antigen can be detected in the blood or serum as early as the prodromal phase of infection, peaking during the acute phase and declining during convalescence. CHB is diagnosed when HBsAg is detected in the serum for a period of six months or longer. ${ }^{23}$

Although HBsAg is the candidate marker for diagnosing HBV infection all around the world, the techniques used in the detection of this marker vary greatly from place to place. In Sri Lanka, the most commonly used test for detecting HBsAg in the clinical setting is either enzyme immunoassays (EIA) or immunochromatography assays (ICA). Comparative studies indicate EIA to be more sensitive and accurate in detecting HBsAg than ICA. ${ }^{24}$ However, ICA is used more frequently than EIA in clinical laboratories in Sri Lanka because it is cheaper and requires less expertise to perform the test. Hence, EIA for HBsAg detection is performed routinely only in reference laboratories and blood banks. The major drawback in using ICA in the detection of HBsAg is that different assays tend to have different accuracy indices which may also differ from one prevailing HBV subtype to another. Chameera et al (2013) ${ }^{24}$ compared two commonly used ICA (CORTEZ'S HBsAg one step and CTK Biotech's Onsite HBsAg) against an EIA (SURASE B-96) to determine their accuracy indices. The study showed that both the ICA (Biotech's Onsite HBsAg assay and CORTEZ'S HBsAg one step assay) were less sensitive (80\% and $97.82 \%$, respectively) and had a low NPV (60\% and $95.74 \%$, respectively) compared to the EIA. ${ }^{24}$ These findings emphasize the need for regular validation of these ICAs with standard methods of detection such as EIA if the ICAs are to be used for routine diagnosis of HBV infection by Sri Lankan laboratories. 


\section{Vaccination and protection against hepatitis B in Sri Lanka}

HBV infection is also an important occupational hazard for health care workers in Sri Lanka based on the risk in certain healthcare settings ${ }^{16}$ and professions ${ }^{13}$. The reasons for this risk at present might be inadequate levels of immunity following vaccination, cost associated with vaccination and testing for immunity.

Vaccination is an easy and cost effective method to prevent HBV infection and associated sequelae. Moreover, vaccination eliminates $\mathrm{HBV}$ infection and reduces chronic carriage, thus limiting the spread of infection to the uninfected. ${ }^{5,6,7}$ As a measure to prevent HBV infection, the Sri Lankan Ministry of Health included Hepatitis B vaccination in the National Immunization Program in Sri Lanka with effect from 2003. ${ }^{25}$ At present, the carrier rate of HBV among the general population in Sri Lanka is not sufficiently high to support state funded immunization of children. Instead, it would be better to continue HBV immunisation to health care workers (HCW) who have an occupational risk. ${ }^{13,16}$ Routine immunization of HCW in the country has been in place for the past twenty years. Anti-HBs levels below $10 \mathrm{mIU} / \mathrm{mL}$ are considered non-responsive to the vaccine and levels above 10 $\mathrm{mIU} / \mathrm{mL}$ are considered as responsive with protective immunity against $\mathrm{HBV}$ infection. ${ }^{26}$

Development of protective immunity in vaccinated individuals depends on a number of factors including age, gender, obesity, alcohol, smoking habits, genetics and pre-existing medical conditions such as malignancy. ${ }^{27}$ Almost $90 \%$ of the individuals below 40 years of age who complete three or two doses of the vaccine acquire protective immunity against HBV infection. ${ }^{27,30}$ The remaining 5 to $10 \%$, remain as non-responders. ${ }^{28}$ However, boosting these individuals result in approximately $50 \%$ of them developing protective immunity. ${ }^{30}$ In individuals over 60 years of age, the development of protective antibody levels reduces to $75 \%$. $^{28}$

Sudies carried to determine the immune response against HBV vaccination in a cohort of HCW in Kandy $^{29}$ and Colombo ${ }^{32}$ also indicate that 9 to $10 \%$ of the vaccinated HCW were non- responsive to the vaccination. The Kandy based study by Chathuranga et al $(2013)^{29}$ also showed that the number of male non-responders were higher than that of the females. This finding too is consistent with studies conducted elsewhere. ${ }^{27,28}$ This difference may be attributed to differences in genetics, nutrition and health. Anti-HBs between 10-100 mIU/mL were found in $24 \%$ of study participants. In the Colombo based study ${ }^{32}$ around $90 \%$ of participants had anti-HBs between $10-100 \mathrm{mIU} / \mathrm{mL}$. In this study booster doses were given to the non-responsive HCW and their antibody titres reassessed. Around $86 \%$ of the boosted individuals developed protective levels of anti-HBs. Nevertheless, a small cohort of HCW remained non-responsive and thus at risk on exposure to $\mathrm{HBV}$. In a more recent study conducted among the HBsAg vaccinated Allied Health Science students $(n=89)$ of the University of Peradeniya, $>98 \%$ of the vaccinees had protective immunity of $>10 \mathrm{mIU} / \mathrm{mL}$ anti-HBs following a single course of immunisation. ${ }^{33}$ This study cohort of all healthy adults between 23-27 years of age supports the positive impact of younger age and health on the production of protective level of anti-HBs in a selected group of vaccinees.

On the other hand, only 310 of 407 (76.2\%) children (aged 1-5 years) vaccinated from the 2003 HBV immunisation initiative showed a protective response $(>10 \mathrm{mIU} / \mathrm{mL}$ anti-HBs $)$. However, a 
waning anti-HBs response with increasing age of these children was also shown in this study. An anti-HBs response of $79.4 \%, 78.6 \%, 76.4 \%$ and $67.9 \%$ have been detected in 1-2, 2-3, 3-4 and 4-5 year olds respectively. ${ }^{34}$ Testing for immunity in children after immunisation is currently not recommended. ${ }^{35}$ Although immunological memory lasts for $>20$ years and an anamnestic anti-HBs response against clinically significant $\mathrm{HBV}$ infection is expected in those who have received a full course of the vaccine in childhood, testing of antibody status on entering healthcare training such as medicine, surgery, dentistry, nursing and laboratory work is recommended to ensure immunity ${ }^{35}$.

\section{Concluding remarks}

Sri Lanka is unique among most developing countries in having a low to intermediate prevalence of HBV infection and thus enjoys a status similar to that of a developed country for HBV infection rates and carriage. High risk groups for contracting HBV infection in Sri Lanka include HCW who have an occupational risk for acquiring the infection and other risk groups such as thalassemia patients undergoing multiple blood transfusions and cancer patients, especially those undergoing invasive tests and therapeutic procedures. Laboratory diagnosis of $\mathrm{HBV}$ infection using $\mathrm{HBs} \mathrm{Ag}$ detection with ICAs has to be done with caution and the assays have to be validated regularly if they are to be used on a regular basis by clinical laboratories. Routine immunization as per the EPI in Sri Lanka has been effective since 2003. Despite routine immunization offered to HCW, low immunity among this population indicates a need for follow up studies to determine protective immunity and re-immunization if necessary. Awareness and educational programmes on HBV transmission are some other measures required to prevent exposure to HBV in the wider community.

Funding: No funding required.

\section{Contributions:}

FN: Conceptualised, wrote and responded to queries from the reviewers and the editor.

FNNP: Wrote certain sections and formatted and contributed in the revision.

RR: Gathered information from the literature.

\section{References}

1. Kane, M. Global programme for control of hepatitis B infection. Vaccine. 1995; 13(S):S47-S49. doi: 10.1016/0264-410X(95)80050-N

2. Noordeen F. Hepatitis B virus infection: An insight into infection outcomes and recent treatment options. Virus Disease 2015; 26: 1-8. doi: 10.1007/s13337-015-0247-y

3. National Expanded Programme on Immunization, Immunization Sri Lanka-2002, Epidemiological Unit, Ministry of Health, Nutrition and Welfare 2002. No doi

4. Epidemiological bulletin fourth quarter 2010.Epidemiological Unit, Ministry of Health 2010; 51. Available at: http://www.epid.gov.lk/web/attachments/article/165/QEB\%204th\%20Quarter.pdf. No doi

5. Liaw YF, Chu CM. Hepatitis B virus infection. Lancet 2009; 373:582-592. doi: 10.1016/S0140-6736(09)60207-5

6. Introduction of hepatitis B vaccine into childhood immunization services, Management guidelines, including information for health workers and parents. WHO 2001 (Unpublished WHO/V\&B/01.31). No doi 
7. World Health Organisation Hepatitis B Fact sheet No 204, WHO 2009. Available at: http://www.who.int/csr/disease/hepatitis. No doi

8. Hepatitis B Information for Health Professionals. CDC 2012. Available at: http://www.cdc.gov/hepatitis/hbv/. No doi

9. World Health Organization. General Alert and Response (GAR) Hepatitis B. WHO 2013. No doi

10. Jilbert, AR, Mason WS, Kann M. Hepatitis B Virus Replication. Hepatitis B virus. S. Locarnini and C. L. Lai. London, Atlanta, Singapore, International Medical Press. 2008; 1: 4.1-4.13. No doi

11. Kim YS, Ahn YO. Factors associated with intrafamilial transmission of hepatitis B virus infection in Korea. Journal of Korean Medical Science 1993; 8:395-404. Available at: http://www.ncbi.nlm.nih.gov/pmc/articles/PMC3053873/. No doi

12. SLMA guidelines and information on vaccines, $4^{\text {th }}$ edition, Sri Lanka Medical Association 2011. Available at: http://issuu.com/slmanews/docs/slma_vaccines_guidelines_2011. No doi

13. Eeswaraarachchige P. A Study on the prevalence of hepatitis B surface antigen carrier status in a district in Sri Lanka and prevalence and risk factors of hepatitis B infection among nursing personnel in the same district. Thesis in MD in Community Medicine, Post Graduate Institute of Medicine, University of Colombo, Sri Lanka, 1993. No doi

14. Premaratne, EDRG. Sero-epidemiology of hepatitis B and C in Colombo District and an estimate of the contribution of these infections in the aetiology of chronic liver diseases. Thesis in MD in Community Medicine, Post Graduate Institute of Medicine, University of Colombo, Sri Lanka, 2002. No doi

15. Gunasena S. Prevalence of hepatitis B virus markers in children with thalassaemia and other diseases requiring multiple blood transfusions. Thesis in MD in Medical Microbiology, Post Graduate Institute of Medicine, University of Colombo, Sri Lanka, 1994. No doi

16. Colombage, GSSK, Hepatitis virus markers among patients treated at the Cancer Institute Maharagama. Thesis in MD in Medical Microbiology, Post Graduate Institute of Medicine, University of Colombo, Sri Lanka, 1996. No doi

17. Lamabadusuriya SP. Immunisation of children: a sound investment for the Millennium. Sri Lanka Journal of Child Health 2000; 29:39-44. No doi

18. Sylva, KKS. Horizontal transmission of hepatitis B virus among household contacts of the asymptomatic paediatric patients at Cancer Institute, Maharagama. Thesis in MD in Medical Microbiology, Post Graduate Institute of Medicine, University of Colombo, Sri Lanka, 2007. No doi

19. Vidanagama D., Colombage GSSK. Prevalence of Hepatitis B Surface antigen carrier state among Antenatal mothers in the Western Province of Sri Lanka. Galle Medical Journal 2010; 16: 1. No doi

20. Editorial. Some thoughts on the revised Sri Lankan expanded programme of immunisation, Sri Lanka. Journal of Child Health 2012; 41(2): 53-55. No doi

21. Manamperi A, Gunawardene NS, Wellawatta C, Abeywickreme W, de Silva HJ. Hepatitis B virus (HBV) genotypes in a group of Sri Lankan patients with chronic infection. Tropical Biomedicine 2011; 28(2):320-324. No doi

22. Jayasuriya DGD, F Noordeen, Pitchai FNN. Genotypes of hepatitis B virus found in patients from a teaching hospital in the central province of Sri Lanka. Ceylon Medical Journal 2015; 60:62-4. doi: http://doi.org/10.4038/cmj.v60i2.8153

23. Krajden M, McNabb G, Petric M. The laboratory diagnosis of hepatitis B virus. Canadian Journal of Infectious Disease and Medical Microbiology 2005; 16:65-72. No doi

24. Chameera EWS., Noordeen F., Pandithasundara H., Abeykoon AMSB. Diagnostic efficacy of rapid assays used for the detection of hepatitis B virus surface antigen. Sri Lankan Journal of Infectious Disease. 2013; 3(2):21-27. doi: http://doi.org/10.4038/sljid.v3i2.5172

25. Immunization hand Book, National Expanded Programme on Immunization, Sri Lanka - 2002, Epidemiological Unit, Ministry of Health, Nutrition and Welfare 2002. Available at: http://www.epid.gov.lk/web/images/pdf/Publication/Immunization_Guide_2012.pdf. No doi 
26. Zuckerman JN, Sabin C, Craig FM et al. Immune response to a new hepatitis B vaccine in healthcare workers who had not responded to standard vaccine: randomised double blind dose-response study. British Medical Journal 1997; 314:329-33. No doi

27. McMahon BJ, Dentinger CM, Burden D et al. Antibody levels and protection after hepatitis B vaccine: Results of a 22-year follow-up study and response to a booster dose. J Infect Dis. 2009; 200:1390-1396. No doi

28. Leuridan E, Van Damme P. Hepatitis B and the need for a booster dose. Clin Infect Dis. 2011:53:6875. No doi

29. Chathuranga LS, Noordeen F, Abeykoon AMSB. Immune response to hepatitis B vaccine in a group of health care workers in Sri Lanka. International Journal of Infectious Diseases 2013; 17:1078-1079. doi: 10.1016/j.ijid.2013.04.009

30. Wood RC, MacDonald KL, White KE, et al.. Risk factors for lack of detectable antibody following hepatitis B vaccination of Minnesota health care workers. Journal of the American Medical Association 1993; 270:2935-72. No doi

31. Westmoreland D, Player V, Heap DC, Hammond A. Immunization against hepatitis B - what can we expect? Results of a survey of antibody response to immunization in persons 'at risk' of occupational exposure to hepatitis B. Epidemiology and Infection 1990; 104:499-509.

No doi

32. Perera J, Perera B, Gamage S. Sero-conversion after hepatitis B vaccination in healthy young adults, and the effect of a booster dose. Ceylon Medical Journal 2002; 47:1. doi: http://doi.org/10.4038/cmj.v47i1.6396w

33. Baddevithana AK, Noordeen F, Mendis CM, Abeykoon AMSB. Immune response to hepatitis B vaccine in a group of vaccinees in the Faculty of Allied Health Sciences of the University of Peradeniya. Sri Lankan Journal of Infectious Diseases. 2015; 5(1):7-12. doi: http://doi.org/10.4038/sljid.v5i1.7994

34. Amarasekara J, Palihawardana P, Peiris S, Galagoda GCS. Assessment of sero-prevalence of hepatitis B antibody and hepatitis B infection after routine immunisation in 1-5 year old children. The Bulletin of the Sri Lanka College of Microbiologists. 2015; 13(1): 11-12. No doi

35. Hepatitis B. A detailed document of the CDC. Accessed $27^{\text {th }}$ of August 2015. Available at: http://www.cdc.gov/vaccines/pubs/pinkbook/downloads/hepb.pdf.

36. Global prevalence of HBsAg positivity, CDC, 2008. Available at: http://wwn.cdc.gov/travel/yellowbookch 4-HepB.aspx. No doi 